\title{
Del concepto formal a la conceptualización sistémica en el diseño arquitectónico.
}

\author{
From formal concept to systemic conceptualization in architectural design.
}

Recibido: agosto 2019

Aceptado: marzo 2020

\section{Resumen}

A pesar de la diversidad de aproximaciones a la conceptualización en el diseño arquitectónico, la idea de que el concepto atiende a una analogía formal está aún extendida. La analogía formal del concepto suele estar vinculada únicamente a aspectos simbólicos de la composición para el observador y no a la esencia de lo descubierto en el proceso de investigación y análisis proyectual. Desde la perspectiva del fenómeno arquitectónico como sistema, esta idea provoca un rompimiento entre la investigación proyectual y la forma, y preserva la dicotomía forma-función. En este artículo se aborda la idea de que el proceso de conceptualización en arquitectura puede ser abordado desde una perspectiva sistémica del fenómeno arquitectónico fundamentada en la noción de autopoiesis de Maturana y Varela, de los sistemas de Christopher Alexander, y la investigación de Flores-Gutiérrez sobre el fenómeno arquitectónico. El texto concluye con la idea de que, al dejar fuera al concepto como guía de composición formal y adoptar un proceso de conceptualización sistémica orientado a la definición de un sistema generador, el diseñador puede ampliar los límites de su investigación para buscar no sólo la configuración y relaciones adecuadas para la actividad, sino la atmósfera necesaria para dichas actividades.

\section{Palabras Clave:}

diseño arquitectónico; sistema; conceptualización; concepto
Avatar Flores Gutiérrez ${ }^{1}$
Despite the diverity of approaches about the conceptualization process in architectural design, the idea behind understanding the concept as a formal analogy in architectural design is still widely spread. The formal analogy of the concept is usually only linked to the symbolical aspects of composition for the observer and not to the essence of the achievements of the research process and the projectual analysis. From the point of view of the architectural phenomenon as a system, this idea prompts a break up between projectual research and form, and preserves the ongoing dichotomy form-function. This paper discusses the idea of that conceptualization process on architecture can be considered as a systemic approach to the architectural phenomenon, based on the notions of autopoiesis developed by Maturana y Varela, Christopher Alexander's systems and the research by FloresGutiérrez on the architectural phenomenon. The text closes with the assumption that discarding the use of the concept as a guide to formal composition and adopting a process of systemic conceptualization oriented to the definition of a generating system, can provide the designer with an expanded field of research to seek not only the right configuration and relationships for activities, but the required atmosphere for those activities.

\section{Keywords:}

architectural design; system; conceptualization; concept

\footnotetext{
${ }^{1}$ Nacionalidad: mexicano; adscripción: profesor e investigador en la Universidad Autónoma de Querétaro. Coordinador de la Maestría en Arquitectura y el Doctorado en Innovación, Tecnología y Hábitat; Doctor en Arquitectura por la Universidad Nacional Autónoma de México; E-mail: avatar.flores@gmail.com
} 


\section{INTRODUCCIÓN.}

"Hablar de arquitectura humana es una evidente tautología, sin embargo, los hechos muestran que es necesario insistir en la función primigenia de la creación de espacios: la satisfacción plena de los usuarios, bajo la premisa: los espacios arquitectónicos y urbanos no deben ser diseñados para ser contemplados, sino para ser vividos cotidianamente, compartiendo con ellos la evolución de nuestro ser"

(Barrios, 2013: 224)

A pesar de la diversidad de aproximaciones sobre la conceptualización en el diseño arquitectónico, la idea de que el concepto atiende a una analogía formal ajena al proyecto está aún extendida. Se practica y se enseña de esta manera. Es común todavía en nuestros días, tanto en aulas como en la práctica profesional, encontrarnos con un concepto formal que hace referencia a un caracol y ver cómo éste se ve reflejado en la forma del edificio que asemeja a un caracol. En algunos casos se trata del volumen completo, en otros de una vista en planta como, por ejemplo, un concepto formal de una paloma de la paz, cuyo edificio, en la vista en planta, tiene esta silueta.

$\mathrm{Su}$ origen, obedece a una natural dialéctica de las ideas sobre el diseño arquitectónico en las cuales había predominado hasta cerca de los años setenta la idea técnica del ensamble de componentes por sobre el valor simbólico (Love, 2003), y que dio origen a una arquitectura que bien podría llamarse conceptual en la que, tal como sucede en el arte conceptual, predominan la importancia de las ideas que subyacen al objeto arquitectónico, por sobre el objeto mismo y la experiencia de habitarlo.

Sin embargo, si ha prevalecido esta tendencia, ha sido en parte debido a una creciente y excesiva valoración de la imagen como medio de transmisión de la arquitectura, que se ha visto acrecentada con los medios electrónicos de comunicación, dejando de lado la experiencia de habitar los espacios. Al respecto, Biondi (2005) relaciona esta tendencia cultural a los medios de difusión de la obra arquitectónica.

"En lo que se refiere a la arquitectura, (...) es siempre más difundida la costumbre de presentar fotografías de obras vacías y deshabitadas (...). En ellas, el culto se ha transferido hacia la imagen misma de la obra, como un objeto supuestamente puro y aislado, acabado en sí mismo. Tales imágenes pueden tal vez comunicar cualidades ideales de la obra, pero nada de su esencia si entendemos ésta como cabal cumplimiento de su doble nexo con el contexto y la finalidad" (Biondi, 2005: 228).

En efecto, uno de los grandes dilemas para la difusión de la obra arquitectónica radica en la imposibilidad de transmitir la experiencia de habitar fuera de su contexto original, tal cual lo haría una pieza musical que es explicada por medio de palabras, o una obra de danza en una sola fotografía, lo que provoca que el foco de atención se centre en lo más evidente y visible que es la forma y la plástica de la obra.

El concepto como analogía formal prevalece también sustentado por la utilidad comercial de la imagen para la venta y promoción de la obra arquitectónica, una imagen devenida en forma que ha sido simplificada de tal suerte que es exaltada como el único valor que es posible que el arquitecto añada al proyecto en arquitectura.

"La forma es la encargada hoy de proyectar sentido al objeto con relación a unos códigos o procesos de codificación que son tácitos en la sociedad de consumo. Será la que nos transmita la calidad del objeto, o lo que es los mismo, el primer paso en su elección. Hemos entrado en una vorágine por su definición y consumo" (Azúa, Felix; citado en Gausa, 2001: 238).

A pesar de que desde hace algunas décadas la idea del concepto formal ha ido perdiendo énfasis en las discusiones teóricas, la academia, y en el trabajo de algunos arquitectos (Love, 2003), es quizá debido a la inercia de procesos de antaño, y a esta exaltación cultural de la imagen, que en la actualidad y, al menos en México, el concepto formal ha prevalecido, sobre todo en la formación de los arquitectos, como el medio fundamental a través del cual el arquitecto se expresa, y para el cual construye simbolismos que, si bien en ocasiones representan el sentir o intereses que se tienen puestos en el proyecto, no son símbolos pensados para el habitante, sino para el observador, una diferencia importante dado que hablamos de dos objetivos distintos: la conformación de la imagen; y la conformación del hábitat humano, siendo este último el lenguaje de la arquitectura y el que es finalmente experimentado por sus habitantes.

Lo simbólico tiene cabida sin lugar a dudas en el proyecto de diseño arquitectónico, más 
no por medio de la imagen como elemento compositivo aislado sino cuando se le considera como componente de la atmósfera del hábitat humano. Desde luego también, cuando ha surgido de un proceso adecuado de conceptualización, la idea del concepto como referente en el proceso de diseño, puede ser sumamente útil si se supone como el camino que puede derivar en la concepción de un espacio habitable, tal como será abordado más adelante.

El problema ocurre cuando la analogía formal del concepto es vinculada únicamente a aspectos simbólicos de la composición para el observador y no a la esencia de lo descubierto en el proceso de conceptualización; una composición que, como ya se ha mencionado, proviene con frecuencia de una primera idea superficial de lo que se busca que represente el edificio para quienes le contemplan (comúnmente en su forma exterior), dejando en segundo plano a sus habitantes.

De lo anterior se desprende una conclusión introductoria que plantea una búsqueda por una aproximación al diseño arquitectónico que, a pesar de las inercias actuales, se aleje del concepto formal creado para el observador y que soporte, en cambio, la vida de sus habitantes, lo cual nos invita a una reflexión sobre la comprensión del proceso de conceptualización en el diseño arquitectónico.

Para adentrarnos en el tema que concierne a la propuesta de distinción entre concepto formal y conceptualización sistémica, es importante señalar que la discusión que se abordará a continuación parte de un paradigma sistémico del diseño arquitectónico y, así mismo, desde una necesaria disociación en la comprensión del valor que adquiere el objeto arquitectónico desde la actividad del diseño arquitectónico, desde su práctica, y no de una perspectiva general de la arquitectura como disciplina. Dicho de otra forma, en este artículo se aborda el diseño arquitectónico desde su consideración como actividad configuradora del espacio habitable, y no desde un enfoque disciplinar, que podría abarcar sus teorías, estilos, valor histórico o artístico, y que podría precisar partir de otros paradigmas de análisis.

Lo que origina pues esta propuesta es la comprensión del ser humano como un ser complejo inmerso en un sistema vital de relaciones que hacen del resultado del diseño arquitectónico en su conjunto, una consecuencia lógica.

\section{EL CONCEPTO EN EL PROCESO DE DISEÑO.}

Apesar de la diversidad de métodos y aproximaciones existentes, en un proceso de diseño arquitectónico podemos generalizar la existencia de dos grandes etapas: Conceptualización (análisis) y desarrollo (síntesis). En la primera surge la comprensión del problema, su caracterización, investigación proyectual, y el proceso de análisis que permitirá en la síntesis la definición de objetivos. La segunda, la de desarrollo, comienza con una fase de diagnóstico en la que se aborda la definición de objetivos, intenciones, criterios y parámetros que habrán de sintetizarse después en el desarrollo o estrategia para la configuración del proyecto de un espacio habitable. Ambas, conceptualización y desarrollo, constituyen la práctica habitual generalizada de un proceso de diseño.

En las dos etapas han existido siempre aspectos que se considera que están supeditados al análisis del contexto y los usuarios, y aspectos que dependen de decisiones de quien proyecta. Por ejemplo, se acepta que el programa arquitectónico depende por completo de aspectos funcionales y de necesidades en la etapa de conceptualización, mientras que se asume un cierto grado de libertad en la forma y la selección de materiales, lo que se traduce en la conformación del concepto formal, como un condicionante adicional externo al problema de diseño.

La confusión parece estar en la comprensión sobre si las decisiones de quien proyecta surgen de su voluntad particular, un ámbito externo al problema de diseño, o si deben surgir también como una consecuencia a partir del mismo análisis del ámbito del problema de diseño. Y para responder a ello, puede ser útil observar someramente la manera en que se enseña el diseño arquitectónico en las escuelas.

El taller ha sido adoptado como la estrategia de enseñanza del diseño arquitectónico, y resulta $\tan$ habitual, que el arquitecto no suele reparar en las particularidades que implica esta forma de enseñanza que, entre otras cosas, es similar a la enseñanza de las artes y los oficios, donde hay un maestro y un aprendiz y donde existen pocos conocimientos generalizables, y se experimenta, en cambio, la manera en que el maestro hace las cosas.

En el taller de diseño arquitectónico se aprenden las técnicas básicas del "oficio", tales como la 
composición, antropometría, organización espacial, dibujo; y se aprehenden también modos de pensar y priorizar las decisiones del proyecto, a través de la imitación de la manera en que el maestro hace las cosas en función de sus propios valores y experiencia, derivando en una enseñanza que cambia sus valores constantemente y cuyos principios dependen de prioridades individuales y no de un fin común, lo que denota un vacío disciplinar en cuanto al proceso de conceptualización, que se ve reflejado en la enseñanza y la práctica de la arquitectura. "Los propios arquitectos discrepan en puntos tan básicos que sus discusiones han de interpretarse como expresión de sus dudas e incertidumbres" (NorbergSchulz, 2008: 10).

Así, entre las fases de conceptualización y desarrollo de los procesos de diseño basados en el concepto formal, irrumpe este tercer elemento añadido, un elemento ajeno, externo, que delata la ausencia de un vínculo lógico y claro entre el análisis y la síntesis del proyecto; entre su conceptualización y posterior desarrollo.

En la figura 1, se muestran de manera generalizada las etapas de un proceso de diseño, y se muestran las ideas bosquejadas en las líneas precedentes sobre los alcances del concepto formal dentro de ese proceso.

No es la intención de este texto discutir sobre la utilidad de la noción del concepto en el proyecto de diseño arquitectónico. Finalmente, en su esencia, el concepto es una forma de pensamiento humano que permite captar los caracteres generales, esenciales de las cosas y de los fenómenos de la realidad objetiva (Rosental y Iudin, 1959).
Un concepto que emerja de un proceso de conceptualización puede volverse desde luego sumamente útil al proyecto arquitectónico, toda vez que representa la síntesis del análisis y la comprensión del fenómeno arquitectónico, sin embargo, en la manera en la que ha sido usado de manera generalizada, es decir, como un concepto formal ajeno, enganchado apenas al conocimiento superficial del fenómeno, nos aleja del proceso de conceptualización que paradójicamente forma los conceptos y los vincula inherentemente a su origen.

\section{LA TÉCNICA PROYECTUAL EN LOS PROCESOS DE DISEÑO.}

Desde principios de este siglo, la técnica ha revolucionado con determinación la etapa de desarrollo en el diseño arquitectónico al incorporar nuevas herramientas, tales como las de dibujo, representación, composición formal, o incluso la fabricación digital, por nombrar algunas (y que aún requieren una evolución congruente con los objetivos del diseño), y en oposición, se ha dejado casi intacta la etapa de conceptualización, aquella que permite abstraer los resultados del análisis y traducirlos a un diagnóstico y la propuesta de objetivos e intenciones concretos. A pesar de haber una inminente evolución en marcha, gracias a la incursión cada vez con más fuerza de otras disciplinas (baste nombrar la psicología ambiental, las incursiones de las neurociencias, la cognición y la psicofísica), y del desarrollo de la teoría misma del diseño arquitectónico, visible en una creciente cantidad de artículos de

Figura 1. Uso del concepto formal en el proceso de diseño

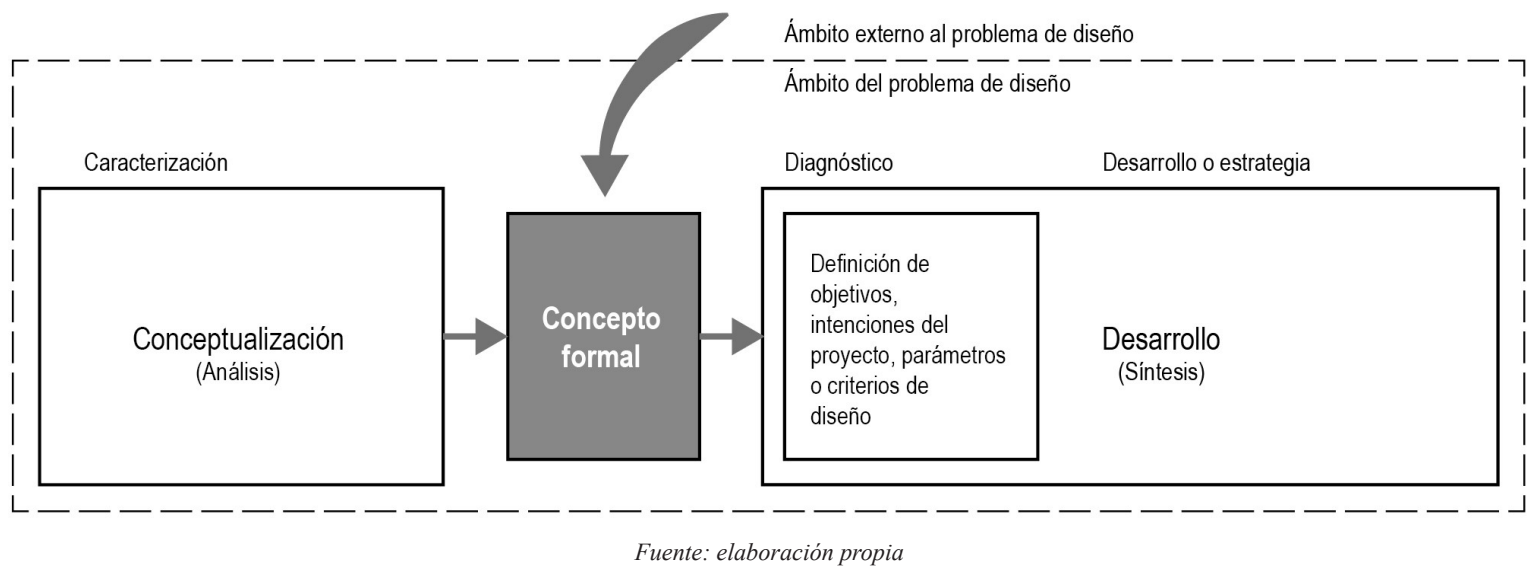


investigación, aún se mantienen en la práctica muchas de las mismas técnicas proyectuales de conceptualización, (llámese análisis de sitio, programa arquitectónico, diagramas de funcionamiento, o concepto formal, por nombrar algunas) que parecen sólo atender los aspectos más técnicos del proyecto.

"El arquitecto ya no puede ser un ser pasivo que resuelva problemas, sino más bien debería aceptar una nueva condición activa, propia de los estrategas, que generan preguntas al entorno y que anticipan líneas de acción. Cuya manera de actuar ya no se basa en la aplicación de un oficio aprendido y consensuado, sino en la innovación de los procesos proyectuales y en la aplicación de nuevas técnicas y materiales que ayuda a desarrollar"

(Gausa et al. 2001: 11)

Al respecto de las herramientas existentes, y evidenciándolas a través de las etapas de la investigación aplicada, oportunas también en los proyectos en arquitectura: caracterización, diagnóstico y estrategia (De la Torre Galindo, 2017), resulta notorio que las técnicas proyectuales de conceptualización existentes apoyen con destreza la caracterización básica del problema, y sin embargo, se carezca de técnicas proyectuales generalizadas respecto a la definición de objetivos e intenciones, propios del diagnóstico, y que corresponden en parte a la síntesis del proyecto, lo que provoca un vacío que ha sido llenado por el concepto formal.

La innovación en procesos proyectuales a la que hace referencia Gausa (2001) es lo que concierne a esta discusión y por la cual se propone que, desde el criterio de una visión sistémica del diseño arquitectónico expuesto en la introducción, es necesaria la generación de nuevas técnicas que apoyen la definición integral del proyecto.

$\mathrm{Si}$ consideramos al espacio arquitectónico desde una perspectiva sistémica del fenómeno arquitectónico (Flores-Gutiérrez, 2016) la idea del concepto formal aislado provoca en primer lugar un rompimiento entre lo observado y analizado en la investigación proyectual y la forma que adquiere, y en segundo, preserva la dicotomía forma-función que no permite articular el proyecto en un todo integral sistémico que responda adecuadamente a la actividad humana entendida en toda su complejidad, tanto física como psicológica. De esta forma, la idea del concepto formal debe ser omitida y asumirse la conceptualización desde la complejidad del sistema vital, y de las posibilidades que nos brindan los conocimientos multidisciplinarios.

Bajo esta premisa, es posible una aproximación integral del problema de diseño arquitectónico, tanto en su conceptualización como en su desarrollo, que posibilite la abstracción del sistema del fenómeno arquitectónico y la comprensión de la conceptualización como un proceso configuracional que abarca todas las dimensiones de la conformación del espacio habitable.

\section{LA VISIÓN SISTÉMICA DEL DISEÑO ARQUITECTÓNICO COMO BASE DE LA CONCEPTUALIZACIÓN.}

"El punto de vista de los sistemas es una versión moderna y disciplinada del sentido de lo maravilloso. Esta es la visión del mundo que asume el hombre cuando toma conciencia de la particularidad y de la totalidad de las cosas del mundo"

(Alexander, 1980: 66)

Sin el afán de profundizar en aspectos que ya han sido discutidos en otros documentos, pero con la intención de aclarar el origen de esta propuesta, es importante establecer brevemente la base y estructura fundamental de la conceptualización sistémica, y que puede entenderse como una visión sistémica del proceso de diseño arquitectónico. Es por ello que se abordan a continuación algunos aspectos de esta visión, que buscan en síntesis establecer algunos principios necesarios para abordar este enfoque.

Los biólogos chilenos, Humberto Maturana y Francisco Varela propusieron la idea de la autopoiesis que, desde su publicación, se desborda de los límites de la biología para instaurarse como una explicación sobre el carácter complejo del sistema vital en el cual está inmerso el ser humano.

En concreto, y respecto al concepto de autopoiesis en los sistemas, para Maturana y Varela:

"Una máquina autopoiética es una máquina organizada como un sistema de procesos de producción de componentes concatenados de tal manera que producen 
componentes que: a) generan los procesos (relaciones) de producción que los producen a través de sus continuas interacciones y transformaciones, y b) constituyen a la máquina como una unidad en el espacio físico"

(Maturana et al. 1994: 69)

Así, la autopoiesis tiene la implicación de que todos los organismos forman parte de un sistema al cual también pertenece el ambiente que habitan. Tanto el ambiente como los mismos organismos configuran su rol (función) y su forma, en función de sus interacciones, es decir, se auto-configuran recíprocamente. La obra arquitectónica es un componente del sistema vital, y como tal, vinculada inherentemente con el proceso que le configura.

El diseño arquitectónico asume entonces el rol de un proceso configurador dentro del sistema vital, un sistema autopoiético que permite la generación de su propia estructura, una estructura congruente con el sistema mismo.

Al hablar de dicho sistema vital, es necesario asumirle como un hábitat humano que, desde la psicología ambiental, puede entenderse como un sistema complejo de relaciones físicas, sociales y psicológicas, y no sólo como uno biológico o ecológico.

(La psicología ambiental) "tiene por objetivo analizar la interrelación entre las personas y los ambientes físicos reales que éstas ocupan. (...) Si bien el foco se centraba (inicialmente) en los ambientes físicos, no tardaron en advertir que a menudo es difícil y artificioso aislar éstos de sus dimensiones sociales, por lo que el objeto de análisis pasó a ser el ambiente o entorno sociofísico"

(Valeri, 2019)

El entorno sociofísico, desde luego sumamente complejo, es el componente ambiental de este gran sistema vital autopoiético.

De la misma forma que Maturana y Varela, Christopher Alexander aborda también la idea de sistema desde sus dos aristas: Como un todo holístico, y como un sistema generador, característica fundamental de todo sistema desde la perspectiva de Alvargonzalez (2016).
"Dos ideas se ocultan en la palabra sistema: la idea de un sistema como un todo y la idea de un sistema generador. (...) Estos dos aspectos, aunque similares, son lógicamente muy distintos. En el primer caso, la palabra sistema se refiere al aspecto particular holístico de una única cosa. En el segundo, la palabra sistema no se refiere en absoluto a una sola cosa, sino a un conjunto de partes y leyes combinatorias en grado de generar muchas cosas"

(Alexander, 1980: 57)

La noción de la conceptualización sistémica radica en la comprensión de que estamos ante un sistema holístico, que es la arquitectura habitada, pero también ante un sistema generador, que es el diseño arquitectónico. Así, el proceso de conceptualización tiene como objetivo la definición del sistema que generará el componente ambiental dentro del propio sistema vital. Es decir, el proyecto en su totalidad surge del análisis, conformando escenarios de actividad $\mathrm{y}$ adquiriendo el rol y la forma inherentes a su participación en el sistema.

"Sin lugar a dudas, el fenómeno arquitectónico se constituye así como un fenómeno sistémico, y de esta forma, todo aspecto o rasgo del operar de sus componentes provienen necesariamente de la dinámica autopoiética del fenómeno"

(Flores-Gutiérrez, 2016: 55)

De esta manera, el rol del proceso de conceptualización es abstraer los aspectos específicos de los componentes del sistema, y sus relaciones.

De lo analizado en las líneas precedentes y en lo que respecta a la visión sistémica del diseño arquitectónico, se pueden establecer los siguientes principios:

- La vida puede ser entendida como un sistema holístico, siendo la arquitectura parte del mismo sistema.

- Bajo una consideración sistémica, el diseño arquitectónico es un proceso configuracional de una de las partes de ese sistema: el espacio habitable.

- $\quad$ El diseño arquitectónico es en sí mismo un sistema generador.

- El proceso de conceptualización debe definir al sistema generador en el diseño arquitectónico. 


\section{LA CONCEPTUALIZACIÓN SISTÉMICA.}

La noción de que el diseño arquitectónico es un proceso configurativo dentro de un gran sistema vital en el cual está inmerso el ser humano y su entorno social y natural emerge de las reflexiones sobre la autopoiesis, los sistemas generativos y la comprensión del fenómeno arquitectónico y explica la diferencia con la idea del concepto formal.

El concepto formal es un determinante externo del proceso de diseño, mientras que la conceptualización sistémica define al sistema generador en el proceso de diseño arquitectónico.

En la figura 2, se aprecia este cambio fundamental respecto a la figura 1. A través de una conceptualización sistémica, el análisis permite un diagnóstico y la definición de objetivos, intenciones, parámetros o criterios de diseño, que deben responder de manera sistémica a lo analizado, de tal manera que el proceso de conceptualización sistémica no se queda sólo en la etapa preliminar de análisis, sino que abarca una parte importantísima del proceso de síntesis del proyecto.

Consecuentemente, aspectos como el complejo sistema de necesidades y satisfactores y la actividad humana compleja, deben ser considerados en el proceso de conceptualización sistémico, mientras que, en la etapa de desarrollo o síntesis, habrán de considerarse aspectos como las capas ambientales que comprende el espacio habitable en el fenómeno arquitectónico.

Así, al considerar el proceso integral de diseño como un proceso configurativo, es indispensable, no sólo un proceso de conceptualización sistémica orientado a la comprensión de los componentes del fenómeno, sino también una síntesis sistémica que responda de tal manera que se mantenga la estabilidad del sistema.

"Las propiedades más importantes de cualquier objeto son aquellas que se relacionan con su estabilidad. (...) La estabilidad en cualquiera de sus formas, es una propiedad holística. Sólo puede ser resultado de una interacción entre partes"

(Alexander, 1980: 60)

La comprensión de esa interacción ocurre también en la conceptualización sistémica, por lo que resulta evidente que la tarea a la que se enfrenta el arquitecto que pretenda abordar el diseño arquitectónico desde esta perspectiva es mayúscula. Se requieren, desde luego, herramientas teóricas e instrumentales que le permitan lograr esta labor. Herramientas que se van haciendo posibles en la medida en que avanza el conocimiento teórico sobre el diseño arquitectónico.

Dados los argumentos expuestos, podemos afirmar que por conceptualización sistémica ha de entenderse el proceso de análisis sobre el sistema del fenómeno arquitectónico ante un problema sociofísico, que contempla la comprensión de las relaciones entre sus componentes con el fin de conformar un sistema generador que permita encontrar la solución espacial arquitectónica que asuma el rol y la forma congruentes con el sistema del que forman parte.

Figura 2. La conceptualización sistémica en el proceso de diseño

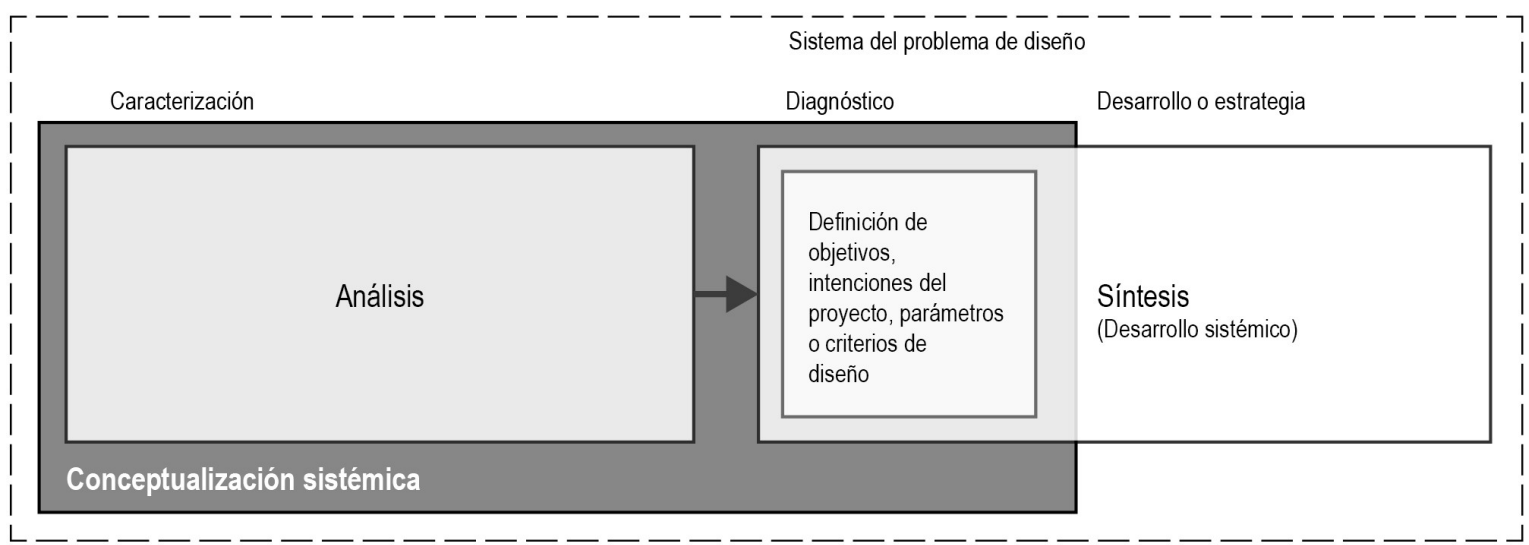

Fuente: elaboración propia 


\section{CONCLUSIONES.}

A partir de la comprensión de la diferencia entre el análisis del diseño arquitectónico y la arquitectura como disciplina, es posible abordar este quehacer desde la visión de un paradigma sistémico desde el cual puede discutirse la pertinencia de la noción de objetivo. El objetivo es relevante dada la definición de sistema de Alvargonzalez (2016), en la cual son necesarios los fines como componente sistematizador de los sistemas propositivos o configuracionales.

Dado lo anterior, es posible definir al diseño arquitectónicocomoun proceso deconceptualización sistémico con fines configuracionales, hecho desde el cual puede cuestionarse la idea del concepto formal como guía compositiva en el diseño arquitectónico.

El concepto formal aislado, como se ha definido en este documento a la idea de que es necesaria una analogía que dé forma al objeto arquitectónico, es una herramienta usualmente ajena a la conceptualización sistémica y la generación de la forma desde la perspectiva del fenómeno arquitectónico (Flores-Gutiérrez, 2016), y suele estar relacionada con una intención vocacional sobre lo que debe representar un edificio a ojos de un observador externo. ¿Es viable definir un concepto formal que en efecto provenga de una conceptualización sistémica? Desde luego, aunque se corre el riesgo de que, al estar tan arraigada esta práctica, se olvide después el origen del mismo concepto y provoque nuevamente una desviación del carácter sistémico del diseño arquitectónico.

Al dejar fuera al concepto como guía de composiciónformal,y adoptarunaconceptualización sistémica, el diseñador puede ampliar los límites de su investigación para buscar no sólo la configuración y relaciones adecuadas para la actividad humana, sino la atmósfera necesaria para dichas actividades, lo que le lleva a una búsqueda formal más exhaustiva y profunda.

El desarrollo de la conceptualización sistémica, y de nuevas herramientas que impulsen su práctica, sólo será posible en tanto se profundice en las bases teóricas del diseño arquitectónico, se transforme también el paradigma de enseñanza del mismo, y se asuma la comprensión del rol del espacio arquitectónico en la vida del ser humano. C

\section{REFERENCIAS.}

Alexander, Christopher (1980). Tres aspectos de matemática y diseño; y la estructura del medio ambiente. Barcelona, Tusquets editores.

Alvargonzalez, David [fgbuenotv] (2016, octubre 26). Las ciencias como sistemas y los sistemas filosóficos [Archivo de video] Recuperado de: http://www.fgbueno.es/ act/efo124.htm

Barrios, Dulce María (2013). El ser humano excluido del diseño del entorno individual y social. En Diego Sánchez González y Carmen Egea Jiménez, Coordinadores. La ciudad, un espacio para la vida, miradas y enfoques desde la experiencia espacial, Ed. Universidad de Granada España pags: 211-225.

Biondi, Stefania (2005). Una visión hermenéutica de la teoría de la arquitectura en México. Tesis doctoral. Programa de maestría y doctorado en arquitectura. Ciudad de México, Universidad Nacional Autónoma de México.

De la Torre Galindo, Francisco Javier, (2017), Recorrer y participar en la ciudad. Aproximación a la adaptación de los recorridos comentados como técnica de la investigación urbana. Ciudad de México: Universidad Autónoma Metropolitana, Unidad Xochimilco, CyAD, Casa y Ciudad A.C., Programa Universitario de Estudios Metropolitanos (PUEM-UAM).

Flores-Gutiérrez, Avatar (2016). Fenómeno arquitectónico, proceso de diseño y complejidad humana: Propuesta de re-conceptualización. Tesis doctoral. Ciudad de México, Universidad Nacional Autónoma de México.

Gausa, Manuel; Guallart, Vicente; Müller, Willi; Soriano, Federico; Morales, José; Porras, Fernando. (2001) Diccionario Metápolis de Arquitectura Avanzada. Ciudad y tecnología en la sociedad de la información. Barcelona, Actar.

Love, Timothy (2003).Kit-of-Parts Conceptualism: Abstracting Architecture in the American Academy. Harvard Design Magazine, No. 19 / Architecture as Conceptual Art? Blurring Disciplinary Boundaries. Cambridge, MA, Harvard University Graduate School of Design. 
Maturana, Humberto; Varela, Francisco (1998). De máquinas y seres vivos. Autopoiesis: la organización de lo vivo. Santiago de Chile, Editorial universitaria.

Norberg-Schulz, Christian (2008). Intenciones en arquitectura. Barcelona, GG Reprints. Editorial Gustavo Gili.

Rosental, M.; Iudin, P. (1959). Diccionariofilosófico abreviado. Montevideo, Ediciones pueblos unidos.

Valera, Sergi; Enric Pol, Tomeu Vidal (2019). Psicología ambiental. Elementos básicos. Departamento de psicología social y psicología cuantitativa. Barcelona, Universidad de Barcelona. Recuperado de [17 de agosto de 2019] http://www.ub.edu/psicologia ambiental/psicologia_ambiental 